\title{
Acknowledgement to Referees
}

Published online: 13 November 2018

(C) Springer Nature Switzerland AG 2018

Dear Reader,

As we reach the final issue of Targeted Oncology for 2018, we wish to reflect on another successful year's achievements, and to thank all those who have contributed their time and effort to guarantee the high quality of our content.

Over 70 articles have been published; the most popular of these, in terms of downloads from SpringerLink, have been:

- Jeffrey M. Clarke et al. Immune Checkpoint Blockade: The New Frontier in Cancer Treatment

- Elvira Schmidt et al. Cabozantinib Versus Standard-of-Care Comparators in the Treatment of Advanced/Metastatic Renal Cell Carcinoma in Treatment-naïve Patients: a Systematic Review and Network Meta-Analysis

- Maximilian Hochmair. Medical Treatment Options for Patients with Epidermal Growth Factor Receptor Mutation-Positive NonSmall Cell Lung Cancer Suffering from Brain Metastases and/or Leptomeningeal Disease

- Shivaani Kummar et al. TRK Inhibition: A New Tumor-Agnostic Treatment Strategy

- Mehran Makvandi et al. Alpha-Emitters and Targeted Alpha Therapy in Oncology: from Basic Science to Clinical Investigations

- Eric Sanchez et al. The Role of B-Cell Maturation Antigen in the Biology and Management of, and as a Potential Therapeutic Target in, Multiple Myeloma

- Smruthi Vijayaraghavan et al. Inhibiting CDK in Cancer Therapy: Current Evidence and Future Directions

- Nadia Khan et al. Targeting BCL-2 in Hematologic Malignancies

- William H. Isacoff et al. Low-Dose Continuous 5-Fluorouracil Combined with Leucovorin, nab-Paclitaxel, Oxaliplatin, and Bevacizumab for Patients with Advanced Pancreatic Cancer: A Retrospective Analysis

- Ofer Purim et al. Biomarker-Driven Therapy in Metastatic Gastric and Esophageal Cancer: Real-Life Clinical Experience

The quality of articles published in the journal is also reflected in its most recent impact factor (3.907). Targeted Oncology is currently ranked 75th in the 'Oncology' category (of 223 journals).

Other journals in the Adis Premier Journals Portfolio (http://www.springer.com/gp/adis) continue to prosper with substantial increases in usage year on year. In addition, this year has seen Applied Health Economics and Health Policy receive its first impact factor.

We thank the authors who have contributed articles to Targeted Oncology over the course of 2018. The skill and dedication of all authors are critical to the continued publication of the journal. The quality of published articles is also testament to the significant efforts of the peer reviewers, whose commitment ensures that the journal's content is held to the highest possible standard. We would like to thank the following individuals who acted as reviewers for Targeted Oncology in the last 12 months: 
Elisabetta Abruzzese, Italy

Thierry Alcindor, Canada

Roberta Alfieri, Italy

Maria Alsina, Spain

Jeanny Aragon-Ching, USA

Hugo Arias-Pulido, USA

Jane Armstrong, UK

Thomas Bachelot, France

Pedro C. Barata, USA

Alexandr Bazhin, Germany

Charlotte Benson, UK

Benoit Beuselinck, Belgium

Neil Bhola, USA

Mehmet Asim Bilen, USA

Cherie Blenkiron, New Zealand

Romain Boidot, France

María Sol Brassesco, Brazil

Adam M. Brufsky, USA

Heiko Bruns, Germany

Laura Carrassa, Italy

Daniel Catenacci, USA

Gieri Cathomas, Switzerland

Anna Chalmers, USA

E. Gabriela Chiorean, USA

Bartosz Chmielowski, USA

James Cleary, USA

Diego Cortinovis, Italy

Padraig D'Arcy, Sweden

Ugo De Giorgi, Italy

Kim De Keersmaecker, Belgium

Adrianus J. de Langen, the Netherlands

Ronald de Wit, the Netherlands

Daniel J. DeAngelo, USA

Marc Denis, France

Neesha Dhani, Canada

Christian Doehn, Germany

Frede Donskov, Denmark

Daniela Dörfel, Germany

Q. Ping Dou, USA

Alexandra Drakaki, USA

Kati Erdmann, Germany

Jérôme Fayette, France

Christos Fountzilas, USA

Daichi Fujimoto, Japan

Masahide Fukudo, Japan

Junji Furuse, Japan

Udo Gaipl, Germany

Timo Gaiser, Germany

Enrique Gallardo, Spain

Hui Gan, Australia

Joan Garrett, USA

Karen A. Gelmon, Canada

Angela George, UK
Sharlene Gill, Canada

Matteo Giulietti, Italy

Ilya G. Glezerman, USA

Simone M. Goldinger, Switzerland

Michael Gough, USA

Amandine Gouverneur, France

Thomas Griffith, USA

Camille Gunderson, USA

Kilian Gust, Austria

John B.A.G. Haanen, the Netherlands

Oliver Hakenberg, Germany

Marcia Hall, UK

Jun Hanaoka, Japan

Mark L. Heaney, USA

Sarah E.M. Herman, USA

Miyoshi Hiroaki, Japan

Vincent Ho, Australia

Paul J. Hoskins, Canada

Eiki Ichihara, Japan

Toshihiko Imamura, Japan

William H. Isacoff, USA

Joon Jeong, Republic of Korea

Rachelle W. Johnson, USA

Oscar Juan, Spain

Ian R. Judson, UK

Solene-Florence Kammerer-Jacquet, France

Niki Karachaliou, Spain

Kyoung-Mee Kim, Republic of Korea

Daniel Krappmann, Germany

Günter Krause, Germany

Thomas Krivak, USA

Ruprecht Kuner, Germany

Athanassios Kyrgidis, Greece

Theodore Laetsch, USA

Christel Larbouret, France

Justin Lathia, USA

Igor Latorzeff, France

Christophe Le Tourneau, France

Michael Lee, Republic of Korea

Karl D. Lewis, USA

Ben Li, USA

Sara López-Tarruella, Spain

Jonathan Loree, Canada

Brigette Buig Yue Ma, Hong Kong

Patrick Ma, USA

Stephan Macher-Goeppinger, Germany

Anantha Marisetty, USA

Maurie Markman, USA

Benjamin Maughan, USA

Felicity May, UK

Andrew L. Mellor, UK

Yasuhiro Miki, Japan

Ryuichi Mizuno, Japan 
Goutam Mondal, USA

Varun Monga, USA

Elena Monti, Italy

Yong Wha Moon, Republic of Korea

Alessandro Morabito, Italy

Deborah Mukherji, Lebanon

Leonhard Müllauer, Austria

Barbara Muz, USA

Sylvie Négrier, France

Tomas Neilan, USA

Carsten Utoft Niemann, Denmark

Maria Carmen Ochoa, Spain

Daniel Olive, France

James F. O'Mahony, Ireland

Egbert Oosterwijk, the Netherlands

Mateusz Opyrchal, USA

Mitsuhiko Osaki, Japan

Tanmay Panchabhai, USA

Elisavet Paplomata, USA

Wendy Parulekar, Canada

Timothy J. Price, Australia

Andrew Protheroe, UK

Ofer Purim, Israel

Suzanne Quartuccio, USA

Guilherme Rabinowits, USA

Luis E. Raez, USA

Jörg Reichrath, Germany

Jin Kyung Rho, Republic of Korea

Richard Riedel, USA

Hanno B. Riess, Germany

Sylvie Rottey, Belgium

Gael Roue, Spain

Hatem Sabaawy, USA

Kazuko Sakai, Japan

Eric Santoni-Rugiu, Denmark

Scott M. Schuetze, USA

Susanne Sebens, Germany

Lorenzo Sempere, USA

Cesar Serrano, Spain
Toshio Shimizu, Japan

Kimihiro Shimizu, Japan

Hiroshi Soda, Japan

Cinzia Solinas, Belgium

Halfdan Sørbye, Norway

Srikala Sridhar, Canada

Birgit Strobl, Austria

Yu Sunakawa, Japan

Shunji Takahashi, Japan

Haruhiko Takeda, Japan

Daniel Taussky, Canada

Diego Tesauro, Italy

Antoine Thiery-Vuillemin, France

Khin Thway, UK

Yusuke Tomita, Japan

Tiffany Troso-Sandoval, USA

Selma Ugurel, Germany

Ulka Vaishampayan, USA

Alexander C.J. van Akkooi, the Netherlands

Michiel van der Heijden, the Netherlands

Anke van Erp, the Netherlands

Nielka van Erp, the Netherlands

Brian van Tine, USA

Anna Varghese, USA

Arndt Vogel, Germany

Derek Wainwright, USA

Eunice Wang, USA

Medhi Wangpaichitr, USA

Andreas Wicki, Switzerland

Jean-Baptiste Woillard, France

Rin Yamaguchi, Japan

Kazuhiro Yamamoto, Japan

Jianming Ying, China

Yoshihito Yokoyama, Japan

Tatsuya Yoshida, Japan

Renato Zambello, Italy

Marjorie G. Zauderer, USA

Viola Zhu, USA

In addition, we would like to thank the members of the journal's Honorary Editorial Board, who have acted as peer reviewers and authors, and have provided guidance on journal content, policy and processes:

Gabriel N. Hortobagyi, Houston, TX, USA

Jean-François Morère, Paris-Nord, France

J.O. Armitage, Omaha, NE, USA

J.R. Berenson, West Hollywood, CA, USA

J.-Y. Blay, Lyon, France

T. Buchler, Prague, Czech Republic

A.-L. Cheng, Taipei, Taiwan

F. Ciardiello, Napoli, Italy

J. Desai, Melbourne, Australia
S. Faivre, Clichy, France

M.M. Fukuoka, Osaka, Japan

G. Giaccone, Washington, DC, USA

F.A. Greco, Nashville, TN, USA

P. Grivas, Seattle, WA, USA

V.C. Jordan, Houston, TX, USA

J.M. Kirkwood, Pittsburgh, PA, USA

H.-J. Lenz, Los Angeles, CA, USA

R. Liang, Hong Kong 
T. Macarulla, Barcelona, Spain W.P. McGuire, Richmond, VA, USA

M.J. McKeage, Auckland, New Zealand

A. mita, Los Angeles, CA, USA

M. Mita, Los Angeles, CA, USA

A.G. Papavassiliou, Athens, Greece

S. Peters, Lausanne, Switzerland

C.C. Porta, Pavia, Italy

S. Qin, Nanjing, China

E. Raymond, Paris, France

N. Reguart, Barcelona, Spain
O. Rixe, Albuquerque, NM, USA

H. Rizos, North Ryde, NSW, Australia

C. Rolfo, Baltimore, MD, USA

H.-J. Schmoll, Halle, Germany

N.A. Shonka, Omaha, NE, USA

D. Soulières, Montréal, Québec, Canada

J. Tabernero, Barcelona, Spain

G. Tortora, Verona, Italy

E. Van Cutsem, Leuven, Belgium

J.E-L. Wong, Singapore

We do hope that you have found the articles published throughout the year in Targeted Oncology to be interesting and informative. The editorial schedule for 2019 is well under way, and we are looking forward to bringing you many high-quality and authoritative articles over the coming year.

With best wishes,

Martin Chopra

Editor

Targeted Oncology 